\title{
DARK MATTER: THE CASE OF STERILE NEUTRINO*
}

\author{
Mikhail Shaposhnikov \\ Institut de Théorie des Phénomènes Physiques, Ecole Polytechnique Fédérale de Lausanne, \\ CH-1015 Lausanne, Switzerland
}

\begin{abstract}
An extension of the Standard Model by three right-handed neutrinos with masses smaller than the electroweak scale (the $\nu \mathrm{MSM}$ ) can explain simultaneously dark matter and baryon asymmetry of the Universe, being consistent with the data on neutrino oscillations. A dark matter candidate in this theory is the sterile neutrino with the mass in $\mathrm{keV}$ range. We discuss the constraints on the properties of this particle and mechanisms of their cosmological production. Baryon asymmetry generation in this model is reviewed. Crucial experiments that can confirm or rule out the $\nu \mathrm{MSM}$ are briefly discussed.
\end{abstract}

\section{Introduction}

There is compelling evidence that the Minimal Standard Model (MSM) of strong and electroweak interactions is not complete. There are several experimental facts that cannot be explained by the MSM. These are neutrino oscillations, the presence of dark matter in the Universe, the baryon asymmetry of the Universe, its flatness, and the existence of cosmological perturbations necessary for structure formation. Indeed, in the MSM neutrinos are strictly massless and do not oscillate. The MSM does not have any candidate for non-baryonic dark matter. Moreover, with the present experimental limit on the Higgs mass, the high-temperature phase transition, required for electroweak baryogenesis, is absent. In addition, it is a challenge to use CP-violation in Kobayashi-Maskawa mixing of quarks to produce baryon asymmetry in the MSM. Finally, the couplings of the single scalar field of the MSM are too large for the Higgs boson to play the role of the inflaton. This means that the MSM is unlikely to be a good effective field theory up to the Planck scale.

$\mathrm{In}^{1-3}$ it was proposed that a simple extension of the MSM by three singlet righthanded neutrinos and by a real scalar field (inflaton) with masses smaller than the electroweak scale may happen to be a correct effective theory up to some highenergy scale, which may be as large as the Planck scale. This model was called "the $\nu$ MSM", underlying the fact that it is the extension of the MSM in the neutrino sector. Contrary to Grand Unified Theories, the $\nu$ MSM does not have any internal

*Based on talks given at 11th Marcel Grossmann Meeting on General Relativity (Berlin, 23.7 29.7.2006), at XXXIII International Conference on High Energy Physics (Moscow, 26.7-2.7.2006), and at 6th International Workshop on the Identification of Dark Matter (Rhodes, 11.9-16.9.2006). To appear in the Proceedings of Marcel Grossmann Meeting. 
hierarchy problem, simply because it is a theory with a single mass scale. Moreover, as the energy behaviour of the gauge couplings in this theory is the same as in the MSM, the absence of gauge-coupling unification in it indicates that there may be no grand unification, in accordance with our assumption of the validity of this theory up to the Planck scale. As well as the MSM, the $\nu$ MSM does not provide any explanation why the weak scale is much smaller than the Planck scale. Similarly to the MSM, all the parameters of the $\nu$ MSM can be determined experimentally since only accessible energy scales are present.

As we demonstrated in, ${ }^{1,2}$ the $\nu$ MSM can explain simultaneously dark matter and baryon asymmetry of the Universe being consistent with neutrino masses and mixings observed experimentally. Moreover, $\mathrm{in}^{3}$ we have shown that inclusion of an inflaton with scale-invariant couplings to the fields of the $\nu \mathrm{MSM}$ allows us to have inflation and provides a common source for electroweak symmetry breaking and Majorana neutrino masses of singlet fermions - sterile neutrinos. The role of the dark matter is played by the lightest sterile neutrino with mass $m_{s}$ in the keV range. In addition, the coherent oscillations of two other, almost degenerate, sterile neutrinos lead to the creation of baryon asymmetry of the Universe ${ }^{2}$ through the splitting of the lepton number between active and sterile neutrinos ${ }^{4}$ and electroweak sphalerons. ${ }^{5}$ For review of other astrophysical applications of sterile neutrinos see talk by Peter Biermann at this conference. ${ }^{6}$

In this talk I review the structure of the $\nu \mathrm{MSM}$ and discuss its dark matter candidate - sterile neutrino. The baryogenesis in this model is briefly reviewed.

\section{The $\nu$ MSM}

If three singlet right-handed fermions $N_{I}$ are added to the Standard Model, the most general renormalizable Lagrangian describing all possible interactions has the form:

$$
L_{\nu M S M}=L_{M S M}+\bar{N}_{I} i \partial_{\mu} \gamma^{\mu} N_{I}-F_{\alpha I} \bar{L}_{\alpha} N_{I} \epsilon \Phi^{*}-\frac{M_{I}}{2} \bar{N}_{I}^{c} N_{I}+\text { h.c. }
$$

where $L_{M S M}$ is the Lagrangian of the MSM, $\Phi$ and $L_{\alpha}(\alpha=e, \mu, \tau)$ are the Higgs and lepton doublets, respectively, and both Dirac $\left(M^{D}=f^{\nu}\langle\Phi\rangle\right)$ and Majorana $\left(M_{I}\right)$ masses for neutrinos are introduced. In comparison with the MSM, the $\nu \mathrm{MSM}$ contains 18 new parameters: 3 Majorana masses of new neutral fermions $N_{i}$, and 15 new Yukawa couplings in the leptonic sector (corresponding to 3 Dirac neutrino masses, 6 mixing angles and $6 \mathrm{CP}$-violating phases).

Let us discuss in general terms what kind of scale for Majorana neutrino masses $M_{I}$ one could expect. If Dirac neutrino masses $\left(M^{D}\right)_{\alpha I}=F_{\alpha I} v$ (where $v=174$ $\mathrm{GeV}$ is the vacuum expectation value of the Higgs doublet) are much smaller than the Majorana masses $M_{I}$, the see-saw formula for active neutrino masses

$$
m_{\nu}=-M^{D} \frac{1}{M_{I}}\left[M^{D}\right]^{T}
$$


is valid. Though it is known that the masses of active neutrinos are smaller than $O(1) \mathrm{eV}$, it is clear that the scale of Majorana neutrino masses cannot be extracted. This is simply because the total number of physical parameters describing $m_{\nu}$ is equal to 9 (three absolute values of neutrino masses, three mixing angles and three $\mathrm{CP}$-violating phases), which is two times smaller than the number of new parameters in the $\nu$ MSM.

A most popular proposal ${ }^{7}$ is to say that the Yukawa couplings $F$ in the activesterile interactions are of the same order of magnitude as those in the quark and charged lepton sector. This choice is usually substantiated by aesthetic considerations, but is not following from any experiment. Then one has to introduce a new energy scale, $M_{I} \sim 10^{10}-10^{15} \mathrm{GeV}$, which may be related to grand unification. The model with this choice of $M_{I}$ has several advantages in comparison with the MSM: it can explain neutrino masses and oscillations, and give rise to baryon asymmetry of the Universe through leptogenesis ${ }^{8}$ and anomalous electroweak number nonconservation at high temperatures. ${ }^{5}$ However, it cannot explain the dark matter as the low energy limit of this theory is simply the MSM with non-zero active neutrino masses coming from dimension five operators. On a theoretical side, as a model with two very distinct energy scales it suffers from a fine-tuning hierarchy problem $M_{I} \gg M_{W}$. Also, since the energy scale which appears in this scenario is so high, it would be impossible to make a direct check of this conjecture by experimental means.

Another suggestion is to fix the Majorana masses of sterile neutrinos in $1-10 \mathrm{eV}$ energy scale ${ }^{9}$ to accommodate the LSND anomaly. ${ }^{10}$ The theory with this choice of parameters, however, cannot explain the baryon asymmetry of the Universe and does not provide a candidate for dark matter particle.

Yet another paradigm is to determine the parameters of the $\nu$ MSM from available observations, i.e. from requirement that it should explain neutrino oscillations, dark matter and baryon asymmetry of the universe in a unified way. It is this choice that will be discussed below. It does not require introduction of any new energy scale, and $M_{I}<M_{W}$. In this case the Yukawa couplings must be much smaller than those in the quark sector, $F<10^{-6}$. The theory has a number of directly testable predictions, which can confirm or reject it.

\section{Dark matter}

Though the $\nu$ MSM does not offer any stable particle besides those already present in the MSM, it contains a sterile neutrino with a life-time exceeding the age of the Universe, provided the corresponding Yukawa coupling is small enough. The decay rate of $N_{1}$ to three active neutrinos and antineutrinos (assuming that $N_{1}$ is the lightest sterile neutrino) is given by

$$
\Gamma_{3 \nu}=\frac{G_{F}^{2} M_{1}^{5} \theta^{2}}{96 \pi^{3}}, \quad \theta=\frac{m_{0}}{M_{1}}, \quad m_{0}^{2}=\sum_{\alpha=e, \mu, \tau}\left|M^{D} \alpha 1\right|^{2},
$$


where $G_{F}$ is the Fermi constant. For example, a choice of $m_{0} \sim O(1) \mathrm{eV}$ and of $M_{1} \sim O(1) \mathrm{keV}$ leads to a sterile neutrino life-time $\sim 10^{17}$ years. ${ }^{11}$

The mass of the sterile dark matter neutrino cannot be too small. An application of the Tremaine-Gunn arguments ${ }^{12}$ to the dwarf spheroidal galaxies ${ }^{13}$ gives the lower bound ${ }^{14} M_{1}>0.3 \mathrm{keV}$. If the sterile neutrino mass is in the $\mathrm{keV}$ region, it may play a role of warm dark matter. ${ }^{15,16}$ Sterile neutrino free streaming length an matter-radiation equality is given by

$$
\lambda_{F S} \simeq 1 \mathrm{Mps}\left(\frac{1 \mathrm{keV}}{M_{1}}\right)\left(\frac{\left\langle p_{s} / T\right\rangle}{3.15}\right)
$$

and the mass inside $\lambda_{F S}$ is

$$
M_{F S} \simeq 3 \times 10^{10} M_{\odot}\left(\frac{1 \mathrm{keV}}{M_{1}}\right)^{3}\left(\frac{\left\langle p_{s} / T\right\rangle}{3.15}\right)^{3},
$$

where $\left\langle p_{s}\right\rangle\left(\left\langle p_{a}\right\rangle\right)$ is an average momentum of sterile (active) neutrino at the moment of structure formation, $M_{\odot}$ is the solar mass. One normally defines cold dark matter $(\mathrm{CDM})$ as that corresponding to $M_{F S}<10^{5} M_{\odot}$, hot DM as the one with $M_{F S}>10^{14} M_{\odot}$, and warm DM as anything in between. Potentially, WDM could solve some problems of the CDM scenario, such as the missing satellites problem ${ }^{17,18}$ and the problem of cuspy profiles in the CDM distributions. ${ }^{19,20}$

Even stronger constraint on the mass of sterile neutrino comes from the analysis of the cosmic microwave background and the matter power spectrum inferred from Lyman- $\alpha$ forest data ${ }^{21,22}: M_{1}>M_{0}\left(\frac{\left\langle p_{s}\right\rangle}{\left\langle p_{a}\right\rangle}\right)$. According to, ${ }^{23} M_{0}=14.5 \mathrm{keV}$, whereas ${ }^{24}$ gives $M_{0}=10 \mathrm{keV}$.

Yet another constraint on the parameters of dark matter sterile neutrino comes from radiative decay $N_{1} \rightarrow \nu \gamma$, suppressed in comparison with $N_{1} \rightarrow 3 \nu$ by a factor $O(\alpha)$ ( $\alpha$ is a fine structure constant). This two body decay produces a line in the spectrum of X-rays coming from dark matter in the Universe; corresponding constraints are discussed in detail in other contributions to these proceedings, see also Refs. ${ }^{25-35}$ To get an idea on admitted Yukawa coupling constants and of Dirac mass of dark matter sterile neutrino see Fig. 1, based on results of Ref. ${ }^{28,32}$

\section{Cosmological production of sterile neutrinos}

Let us discuss now cosmological production of sterile neutrinos. In the region of the parameter space admitted by X-ray observations sterile neutrinos were never in thermal equilibrium in the early Universe. ${ }^{15}$ This means that their abundance cannot be predicted in the framework of the $\nu \mathrm{MSM}:{ }^{36}$ one should either fix the concentration of sterile neutrinos at temperatures greater than $1 \mathrm{GeV}$, or specify the physics beyond the $\nu$ MSM.

One can address the question how many sterile neutrinos are produced due to the $\nu \mathrm{MSM}$ interactions, eq. (1), i.e. because of the mixing with active neutrino flavours 


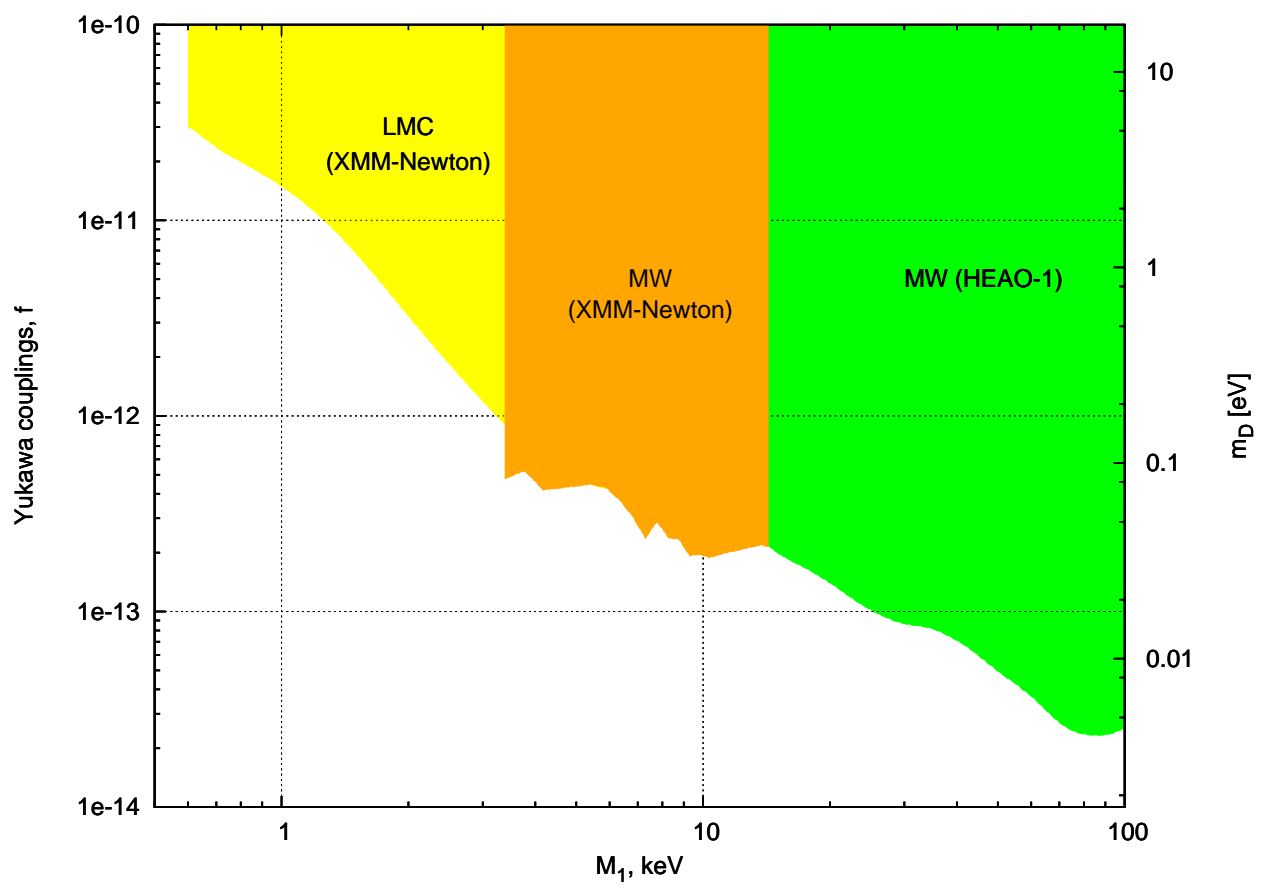

Fig. 1. Upper bound on Yukawa coupling constant (left vertical axis) and Dirac mass (right vertical axis) of dark matter sterile neutrino, coming from X-ray observations of Large Magellanic Cloud (LMC) and Milky Way (MW) by XMM-Newton and HEAO-1 satellites.

characterized by parameter $\theta$. In fact, this mixing is temperature dependent: ${ }^{37}$

$$
\theta \rightarrow \theta_{M} \simeq \frac{\theta}{1+2.4(T / 200 \mathrm{MeV})^{6}\left(\mathrm{keV} / \mathrm{M}_{1}\right)^{2}},
$$

so that the rate $\Gamma$ of sterile neutrino production is strong suppressed at $T>100$ $\mathrm{MeV}, \Gamma \propto T^{-7}$. The rate peaks roughly at ${ }^{15} T_{\text {peak }} \sim 130\left(\frac{M_{I}}{1 \mathrm{kVV}}\right)^{1 / 3} \mathrm{MeV}$, which corresponds to the temperature of the QCD cross-over for keV scale sterile neutrinos. This fact makes an exact estimate of the number of produced sterile neutrinos to be a very difficult task ( $\mathrm{se}^{36}$ for a discussion of the general formalism for computation of sterile neutrino abundance), since $T_{\text {peak }}$ happens to be exactly at the point where the quark-gluon plasma is strongly coupled and the dilute hadron gas picture is not valid. The chiral perturbation theory works only at $T<50 \mathrm{MeV}$. The perturbation theory in QCD works only at $T \gg \Lambda_{Q C D}$, and the convergence is very slow. The lattice simulations work very well for pure gluodynamics. However, no results with three light quarks and with reliable extrapolation to continuum limit are available yet. Also, the treatment of hadronic initial and final states in reactions $\nu+q \rightarrow \nu+q, q+\bar{q} \rightarrow \nu \bar{\nu}$ is quite uncertain. In refs. ${ }^{11,15}$ the computation of sterile neutrino production was done with the use of simplified kinetic equations and without accounting for hadronic degrees of freedom. $\operatorname{In}^{16,38}$ some effects related 
to existence of quarks and hadrons in the media were included; the same type of kinetic equations were used. $\mathrm{In}^{39}$ a computation of sterile neutrino production based on first principles of statistical physics and quantum field theory has been done and uncertainties related to hadronic dynamics were analyzed. The results are presented in Fig. 2. They correspond to the case when there is no entropy production $(S=1)$ due to decay of heavier sterile neutrinos of the $\nu$ MSM. ${ }^{40}$ The area above dotted line is certainly excluded: the amount of produced dark matter would lead to over-closer of the universe. The region below dashed line is certainly allowed: the amount of sterile neutrinos produced due to active-sterile transitions is smaller than the amount of dark matter observed. Any point in the region between two solid lines (corresponding to the "most reasonable" model for hadronic contribution ${ }^{39}$ ) can lead to dark matter generation entirely due to active-sterile transitions. Maximal variation of the hadronic model, defined in ${ }^{39}$ extends this region to the space between dotted and dashed lines. In the case of entropy production with $S>1$ all these four lines simply move up by a factor $S$.

One can see that the active-sterile mixing can accommodate for all dark matter only if $M_{1}<3.5 \mathrm{keV}$, if the "most reasonable" hadronic model is taken. The most conservative limit would correspond to $M_{1}<6 \mathrm{keV}$, if all hadronic uncertainties are pushed in the same direction and the uncertainty by a factor of 2 is admitted for the X-ray bounds. Therefore, if Lyman- $\alpha$ constraints of ${ }^{23,24}$ are taken for granted, the production of sterile neutrinos due to active-sterile neutrino transitions happens to be too small to account for observed abundance of dark matter. In other words, physics beyond the $\nu \mathrm{MSM}$ is likely to be required to produce dark matter sterile neutrinos. Another option is to assume that the universe contained relatively large lepton asymmetries. ${ }^{41}$

$\mathrm{In}^{3}$ it was proposed the the $\nu \mathrm{MSM}$ may be extended by a light inflaton in order to accommodate inflation. To reduce the number of parameters and to have a common source for the Higgs and sterile neutrino masses the inflaton- $\nu$ MSM couplings can be taken to be scale invariant on the classical level:

$$
\mathcal{L}_{\nu \mathrm{MSM}} \rightarrow \mathcal{L}_{\nu \mathrm{MSM}[\mathrm{M} \rightarrow 0]}+\frac{1}{2}\left(\partial_{\mu} \chi\right)^{2}-\frac{f_{I}}{2} \bar{N}_{I}^{c} N_{I} \chi+\text { h.c. }-\mathrm{V}(\Phi, \chi),
$$

where the Higgs-inflaton potential is given by:

$$
V(\Phi, \chi)=\lambda\left(\Phi^{\dagger} \Phi-\frac{\alpha}{\lambda} \chi^{2}\right)^{2}+\frac{\beta}{4} \chi^{4}-\frac{1}{2} m_{\chi}^{2} \chi^{2}
$$

The requirement that the chaotic inflation ${ }^{42}$ produces the correct amplitude for scalar perturbations leads to the constraints:

$$
\beta \simeq 10^{-13}, \quad \alpha \lesssim 10^{-7}, \quad f_{I} \lesssim 10^{-3} .
$$

For $\alpha>\beta$ inflaton mass is smaller than the Higgs mass, $m_{I}<M_{H}$.

One can show ${ }^{3}$ that the inflaton with mass $m_{I}>300 \mathrm{MeV}$ is in thermal equilibrium thanks to reactions $\chi \leftrightarrow e^{+} e^{-}, \chi \leftrightarrow \mu^{+} \mu^{-}$down to $T<m_{I}$. The sterile 


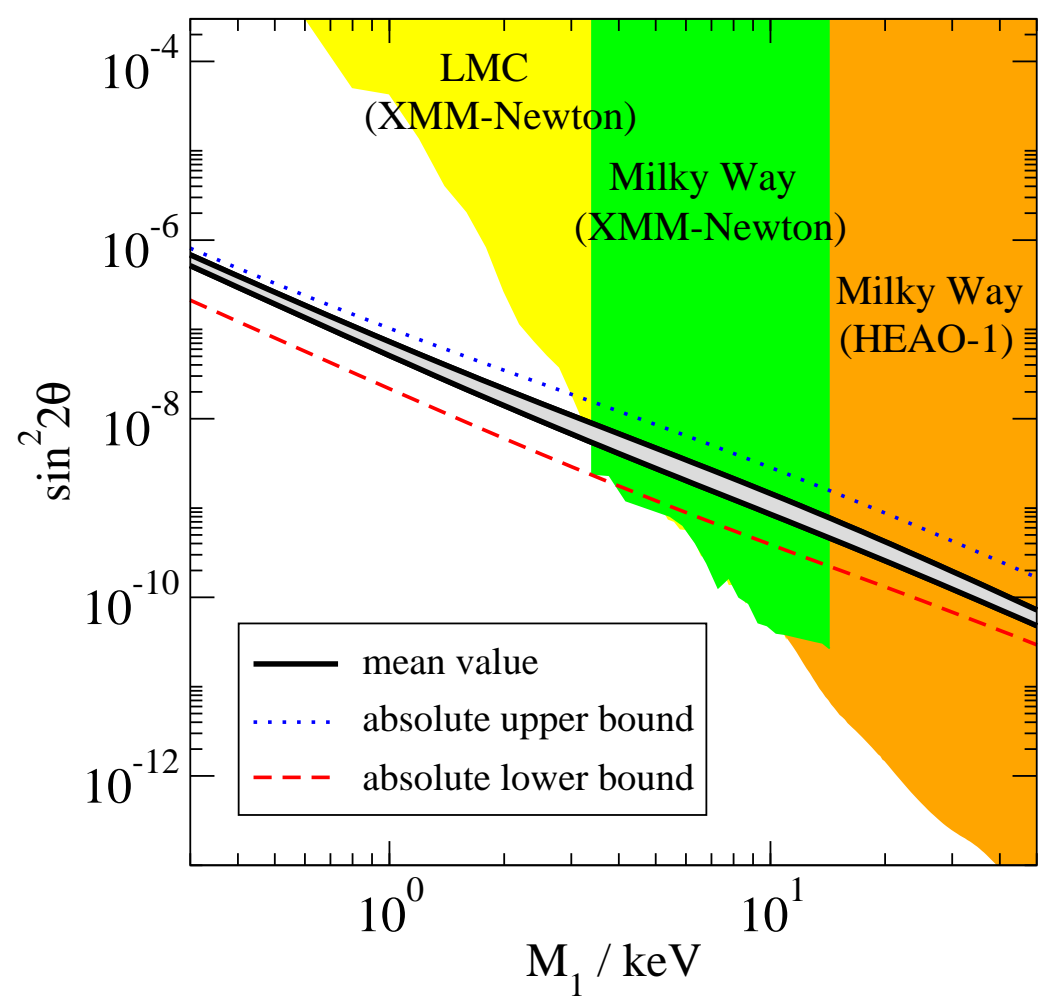

Fig. 2. X-ray constraints from ${ }^{28,32}$ versus required mixing of sterile neutrino in Dodelson-Widrow scenario. It is assumed that no entropy production from decays of heavier sterile neutrinos of the $\nu \mathrm{MSM}$ is taking place. The area between two solid lines corresponds to all possible variations of mixing angles to different leptonic families for "best choice" hadronic dynamics. ${ }^{39}$ The area between dotted and dashed lines corresponds to most conservative estimate of hadronic uncertainties. ${ }^{39}$

neutrino abundance due to inflaton decays: $\chi \rightarrow N N$ is given by

$$
\Omega_{s} \simeq 0.26 \frac{\Gamma M_{0} m_{s}}{m_{I}^{2} \times 12 \mathrm{eV}} \frac{2 \pi \zeta(5)}{\zeta(3)} .
$$

So, for $m_{I} \sim 300 \mathrm{MeV}\left(m_{I} \sim 100 \mathrm{GeV}\right)$ the correct $\Omega_{s}$ is obtained for $m_{s} \sim 16-20$ $\mathrm{keV}\left(m_{s} \sim \mathcal{O}(10) \mathrm{MeV}\right)$. A sterile neutrino in this mass range is perfectly consistent with all cosmological and astrophysical observations. As for the bounds on mass versus active-sterile mixing coming from X-ray observations of our galaxy and its dwarf satellites, they are easily satisfied since the production mechanism of sterile neutrinos discussed above has nothing to do with the active-sterile neutrino mixing leading to the radiative mode of sterile neutrino decay.

\section{Baryon Asymmetry of the Universe}

The baryon (B) and lepton (L) numbers are not conserved in the $\nu$ MSM. The lepton number is violated by the Majorana neutrino masses, while $B+L$ is broken by 
the electroweak anomaly. As a result, the sphaleron processes with baryon number non-conservation are in thermal equilibrium for $100 \mathrm{GeV}<T<10^{12} \mathrm{GeV}$. As for CP-breaking, the $\nu \mathrm{MSM}$ contains $6 \mathrm{CP}$-violating phases in the lepton sector and a Kobayashi-Maskawa phase in the quark sector. This makes two of the Sakharov conditions $^{43}$ for baryogenesis satisfied. Similarly to the MSM, this theory does not have an electroweak phase transition with allowed values for the Higgs mass, ${ }^{44}$ making impossible the electroweak baryogenesis, associated with the non-equilibrium bubble expansion. However, the $\nu$ MSM contains extra degrees of freedom - sterile neutrinos - which may be out of thermal equilibrium exactly because their Yukawa couplings to ordinary fermions are very small. The latter fact is a key point for the baryogenesis in the $\nu \mathrm{MSM}$, ensuring the validity of the third Sakharov condition.

$\mathrm{In}^{4}$ it was proposed that the baryon asymmetry can be generated through CPviolating sterile neutrino oscillations. For small Majorana masses the total lepton number of the system, defined as the lepton number of active neutrinos plus the total helicity of sterile neutrinos, is conserved and equal to zero during the Universe's evolution. However, because of oscillations the lepton number of active neutrinos becomes different from zero and gets transferred to the baryon number due to rapid sphaleron transitions. Roughly speaking, the resulting baryon asymmetry is equal to the lepton asymmetry at the sphaleron freeze-out.

The kinetics of sterile neutrino oscillations and of the transfers of leptonic number between active and sterile neutrino sectors has been worked out in. ${ }^{2}$ The effects to be taken into account include oscillations, creation and destruction of sterile and active neutrinos, coherence in sterile neutrino sector and its lost due to interaction with the medium, dynamical asymmetries in active neutrinos and charged leptons. The corresponding equations are written in terms of the density matrix for sterile neutrinos and concentrations of active neutrinos and are rather lengthy and will not be presented here due to the lack of space. They can be found in the original work. $^{2}$ The corresponding equations are to be solved with the choice of the $\nu \mathrm{MSM}$ parameters consistent with the experiments on neutrino oscillations and with the requirement that dark matter neutrino has the necessary properties.

The value of baryon to entropy ratio $\frac{n_{B}}{s}$ can be found from the solution of the kinetic equations and is given $b^{2}$

$$
\frac{n_{B}}{s} \simeq 1.7 \cdot 10^{-10} \delta_{\mathrm{CP}}\left(\frac{10^{-5}}{\Delta M_{32}^{2} / M_{3}^{2}}\right)^{\frac{2}{3}}\left(\frac{M_{3}}{10 \mathrm{GeV}}\right)^{\frac{5}{3}}
$$

where $M_{2,3}$ are the masses of the heavier sterile neutrinos, $\Delta M_{32}^{2}=M_{3}^{2}-M_{2}^{2}$, and the CP-breaking factor $\delta_{\mathrm{CP}}$ is expressed through the different mixing angles and $\mathrm{CP}$-violating phases, parameterizing the Dirac neutrino masses, and can be $\mathcal{O}(1)$, given the present experimental data on neutrino oscillations. This shows that the correct baryon asymmetry of the Universe $\frac{n_{B}}{s} \simeq(8.8-9.8) \times 10^{-11}$ is generated when the heavier sterile neutrinos with the masses, say, $1 \mathrm{GeV}$ are degenerate to one part in $10^{5}$. This looks like a strong fine tuning but may also indicate that there exists some symmetry making the degeneracy automatic. ${ }^{45}$ 
It is interesting to note that for masses of sterile neutrinos $>100 \mathrm{GeV}$ the mechanism does not work as the sterile neutrinos equilibrate. Also, the temperature of baryogenesis is rather low, $T_{L} \simeq\left(\Delta M^{2} M_{P l}\right)^{\frac{1}{3}}>10^{2} \mathrm{GeV}$, i.e. validity of $\nu \mathrm{MSM}$ is only required at the scales smaller than $M_{W}$ or so.

\section{Conclusions}

The $\nu$ MSM is, perhaps, the simplest and the most economical extension of the Minimal Standard Model. It shares with the MSM its advantages (renormalizability and agreement with most particle physics experiments) and its fine-tuning problems (the gauge hierarchy problem, flavour problem, etc). However, unlike the MSM, the $\nu \mathrm{MSM}$ can explain simultaneously three different phenomena, observed experimentally, namely neutrino oscillations, dark matter, and baryon asymmetry of the Universe. The parameter-space of the model is rather constrained: the dark matter neutrino should have a mass in the keV region and be much lighter than two heavier sterile neutrinos, which are required to be quite degenerate. The model has a number of testable predictions. In astrophysics, one should search for X-rays from decays of dark matter neutrinos, which could be achieved with a X-ray spectrometer in Space with good energy resolution $\delta E / E \sim 10^{-3}-10^{-4}$ getting signals from our Galaxy and its Dwarf satellites. ${ }^{28,35}$ In particle physics, the $\nu$ MSM predicts the absolute values of active neutrino masses, ${ }^{1,46}$ and existence of relatively light singlet fermions ${ }^{45}$ which can be searched for in decays of charmed, beauty and even $K$ or $\pi$-mesons in experiments similar to. ${ }^{47,48}$ The dark matter neutrino can be looked for in $\beta$-decays of tritium and other isotopes. ${ }^{49}$

\section{Acknowledgments}

This work was supported in part by the Swiss National Science Foundation. It is a pleasure to thank Takehiko Asaka, Fedor Bezrukov, Steve Blanchet, Alexey Boyarsky, Alexander Kusenko, Mikko Laine, Andrei Neronov, Oleg Ruchayskiy, and Igor Tkachev for collaboration.

\section{References}

1. T. Asaka, S. Blanchet and M. Shaposhnikov, Phys. Lett. B 631 (2005) 151.

2. T. Asaka and M. Shaposhnikov, Phys. Lett. B 620 (2005) 17.

3. M. Shaposhnikov and I. Tkachev, Phys. Lett. B 639 (2006) 414.

4. E. Akhmedov, V. Rubakov and A. Smirnov, Phys. Rev. Lett. 81 (1998) 1359.

5. V. A. Kuzmin, V. A. Rubakov and M. E. Shaposhnikov, Phys. Lett. B 155 (1985) 36.

6. P. L. Biermann and F. Munyaneza, arXiv:astro-ph/0702173.

7. P. Minkowski, Phys. Lett. B 67 (1977) 421; T. Yanagida, Progr. Theor. Phys. 64 (1980) 1103 ; M. Gell-Mann, P. Ramond and R. Slansky, in Supergravity, North Holland, Amsterdam 1980.

8. M. Fukugita and T. Yanagida, Phys. Lett. B 174 (1986) 45.

9. A. de Gouvea, Phys. Rev. D 72 (2005) 033005.

10. A. Aguilar et al. [LSND Collaboration], Phys. Rev. D 64 (2001) 112007. 
11. A. D. Dolgov and S. H. Hansen, Astropart. Phys. 16 (2002) 339.

12. S. Tremaine and J. E. Gunn, Phys. Rev. Lett. 42 (1979) 407.

13. D. N. C. Lin and S. M. Faber, Astrophys. J. 266 (1983) L21.

14. J. J. Dalcanton and C. J. Hogan, Astrophys. J. 561 (2001) 35.

15. S. Dodelson and L. M. Widrow, Phys. Rev. Lett. 72 (1994) 17.

16. K. Abazajian, G. M. Fuller and M. Patel, Phys. Rev. D 64 (2001) 023501.

17. B. Moore et al., Astrophys. J. 524 (1999) L19.

18. P. Bode, J. P. Ostriker and N. Turok, Astrophys. J. 556 (2001) 93.

19. T. Goerdt et al., Mon. Not. Roy. Astron. Soc. 368 (2006) 1073.

20. G. Gilmore et al., arXiv:astro-ph/0608528.

21. S.H. Hansen, J. Lesgourgues, S. Pastor and J. Silk, Mon. Not. Roy. Astron. Soc. 333 (2002) 544.

22. M. Viel, J. Lesgourgues, M.G. Haehnelt, S. Matarrese and A. Riotto, Phys. Rev. D71 (2005) 063534.

23. U. Seljak, A. Makarov, P. McDonald and H. Trac, Phys. Rev. Lett. 97 (2006) 191303.

24. M. Viel, J. Lesgourgues, M.G. Haehnelt, S. Matarrese and A. Riotto, Phys. Rev. Lett. 97 (2006) 071301.

25. K. Abazajian, G. M. Fuller and W. H. Tucker, Astrophys. J. 562 (2001) 593.

26. A. Boyarsky, A. Neronov, O. Ruchayskiy and M. Shaposhnikov, Mon. Not. Roy. Astron. Soc. 370 (2006) 213.

27. A. Boyarsky, A. Neronov, O. Ruchayskiy and M. Shaposhnikov, Phys. Rev. D74 (2006) 103506.

28. A. Boyarsky, A. Neronov, O. Ruchayskiy, M. Shaposhnikov and I. Tkachev, Phys. Rev. Lett. 97 (2006) 261302.

29. S. Riemer-Sorensen, S. H. Hansen and K. Pedersen, Astrophys. J. 644 (2006) L33.

30. C. R. Watson, J. F. Beacom, H. Yuksel and T. P. Walker, Phys. Rev. D 74 (2006) 033009 .

31. S. Riemer-Sorensen, K. Pedersen, S. H. Hansen and H. Dahle, astro-ph/0610034.

32. A. Boyarsky, J. Nevalainen and O. Ruchayskiy, astro-ph/0610961.

33. A. Boyarsky, O. Ruchayskiy and M. Markevitch, astro-ph/0611168.

34. K. N. Abazajian, M. Markevitch, S. M. Koushiappas and R. C. Hickox, arXiv:astro$\mathrm{ph} / 0611144$.

35. A. Boyarsky, J. W. d. Herder, A. Neronov and O. Ruchayskiy, arXiv:astro-ph/0612219.

36. T. Asaka, M. Laine and M. Shaposhnikov, JHEP 0606 (2006) 053.

37. R. Barbieri and A. Dolgov, Phys. Lett. B 237 (1990) 440.

38. K. Abazajian, Phys. Rev. D 73 (2006) 063513.

39. T. Asaka, M. Laine and M. Shaposhnikov, JHEP 0701 (2007) 091.

40. T. Asaka, M. Shaposhnikov and A. Kusenko, Phys. Lett. B 638 (2006) 401.

41. X. Shi and G.M. Fuller, Phys. Rev. Lett. 82 (1999) 2832.

42. A. D. Linde, Phys. Lett. B 129 (1983) 177.

43. A. D. Sakharov, Pisma ZhETF 5 (1967) 32

44. K. Kajantie, M. Laine, K. Rummukainen and M. E. Shaposhnikov, Phys. Rev. Lett. 77 (1996) 2887.

45. M. Shaposhnikov, Nucl. Phys. B 763 (2007) 49.

46. A. Boyarsky, A. Neronov, O. Ruchayskiy and M. Shaposhnikov, JETP Lett. 83 (2006) 133.

47. G. Bernardi et al., Phys. Lett. B 203 (1988) 332.

48. P. Astier et al. [NOMAD Collaboration], Phys. Lett. B 506 (2001) 27.

49. F. Bezrukov and M. Shaposhnikov, Phys. Rev. D 75 (2007) 053005. 